\title{
Sensitivity of remotely sensed trace gas concentrations to polarisation
}

\author{
D. M. O'Brien ${ }^{1}$, I. N. Polonsky ${ }^{2}$, and J. B. Kumer ${ }^{3}$ \\ ${ }^{1}$ Greenhouse Gas Monitor Australia Pty Ltd, Melbourne, Australia \\ ${ }^{2} \mathrm{RT}$ and RS Solutions, Tucson, USA \\ ${ }^{3}$ Advanced Technology Center, Lockheed-Martin, Palo Alto, USA \\ Correspondence to: D. M. O’Brien (denis.obrien@ggma.com.au)
}

Received: 5 June 2015 - Published in Atmos. Meas. Tech. Discuss.: 24 August 2015

Revised: 29 October 2015 - Accepted: 29 October 2015 - Published: 23 November 2015

\begin{abstract}
Current and proposed space missions estimate column-averaged concentrations of trace gases $\left(\mathrm{CO}_{2}, \mathrm{CH}_{4}\right.$ and $\mathrm{CO}$ ) from high resolution spectra of reflected sunlight in absorption bands of the gases. The radiance leaving the top of the atmosphere is partially polarised by both reflection at the surface and scattering within the atmosphere. Generally, the polarisation state is unknown and could degrade the accuracy of the concentration measurements. The sensitivity to polarisation is modelled for the proposed geoCARB instrument, which will include neither polarisers nor polarisation scramblers to select particular polarisation states from the incident radiation. The radiometric and polarimetric calibrations proposed for geoCARB are outlined, and a model is developed for the polarisation properties of the geoCARB spectrographs. This model depends principally upon the efficiencies of the gratings to polarisations parallel and perpendicular to the rulings of the gratings. Next, an ensemble of polarised spectra is simulated for geoCARB observing targets in India, China and Australia from geostationary orbit at longitude $110^{\circ} \mathrm{E}$. The spectra are analysed to recover the trace gas concentrations in two modes, the first denied access to the polarimetric calibration and the second with access. The retrieved concentrations using the calibration data are almost identical to those that would be obtained with polarisation scramblers, while the retrievals without calibration data contain outliers that do not meet the accuracies demanded by the mission.
\end{abstract}

\section{Introduction}

The Greenhouse gases Observing SATellite (GOSAT) launched by Japan's Aerospace Exploration Agency estimates column-averaged concentrations ${ }^{1}$ of $\mathrm{CO}_{2}$ and $\mathrm{CH}_{4}$ from high resolution spectra of reflected sunlight in absorption bands of $\mathrm{CO}_{2}, \mathrm{CH}_{4}$ and $\mathrm{O}_{2}$. Similarly, NASA's second Orbiting Carbon Observatory (OCO-2) estimates $\mathrm{CO}_{2}$ from $\mathrm{CO}_{2}$ and $\mathrm{O}_{2}$ spectra. While GOSAT measures two orthogonal polarisations, OCO-2 measures only one. In contrast, geoCARB (Sawyer et al., 2013; Mobilia et al., 2013; Kumer et al., 2013; Polonsky et al., 2014; Rayner et al., 2014), proposed to measure $\mathrm{CO}_{2}, \mathrm{CH}_{4}$ and $\mathrm{CO}$ from a geostationary platform, will have inherent sensitivity to polarisation, principally through the diffraction gratings, but will not have any hardware (like GOSAT) or adopt any flight manoeuvres (like OCO-2) to select specific polarisations. The question arises as to whether the sensitivity of the instrument to polarisation causes significant error in retrieved gas concentrations.

This paper uses the following methodology to address this issue. First, in Sect. 2 a model is developed for the polarising properties of the geoCARB spectrographs. The model depends on parameters characterising the optics and the potentially non-linear responses of the detectors; the procedure by which these parameters will be determined during preflight calibration of geoCARB is outlined in Sect. 3. A simplified model that requires only the absolute efficiencies of the gratings is described in Sect. 4.

\footnotetext{
${ }^{1}$ The term concentration is used here in its common English language sense. More precisely we mean dry-air mole fractions.
} 
Next a numerical simulator is flown over a model world to generate an ensemble of polarised spectra that captures much of the variability seen in the real world. For each spectrum in the ensemble, the Stokes vector is computed at the entrance aperture of geoCARB above the atmosphere, and the intensities falling upon the detectors are simulated using the simplified model. For these simulations, described in Sect. 5, geo$\mathrm{CARB}$ is assumed to be at longitude $110^{\circ} \mathrm{E}$ and three frames of data are considered. The first is centred on Agra in India $\left(27.18^{\circ} \mathrm{N}, 78.02^{\circ} \mathrm{E}\right)$, and consists of 1001 pixels observed simultaneously in the $4 \mathrm{~s}$ integration time of geoCARB. The pixels are aligned approximately north-south, and include ocean in the south and the Himalaya in the north. The second and third frames, similarly consisting of 1001 pixels, are centred on Wuhan in China $\left(30.35^{\circ} \mathrm{N}, 114.17^{\circ} \mathrm{E}\right)$ and $\mathrm{Al}-$ ice Springs in Australia $\left(23.42^{\circ} \mathrm{S}, 133.52^{\circ} \mathrm{E}\right)$. In order to include a variety of illumination and observation geometries, each frame is sampled three times per day, the first 3 hours before solar noon, the second at solar noon, and the third 3 hours after. Four days are simulated close to the solstices and equinoxes.

In Sect. 6 the simulated signals, computed taking into account the polarising properties of the surface, clouds, aerosols and molecules, are passed to the inversion algorithm that estimates the column-averaged concentrations of $\mathrm{CO}_{2}$, $\mathrm{CH}_{4}$ and $\mathrm{CO}$, respectively denoted $X_{\mathrm{CO}_{2}}, X_{\mathrm{CH}_{4}}$ and $X_{\mathrm{CO}}$. The inversion algorithm is denied access to the polarising properties of the surface and the atmosphere. Instead it assumes that the surface is Lambertian and non-polarising, but it generates polarising elements internally as it allocates and distributes clouds and aerosols while attempting to match its prediction of the intensity incident upon the detector with the "true" intensity from the simulator. The source of polarisation within the retrieval algorithm is via scattering by clouds, aerosols and molecules. Statistics of the differences between the retrieved and true concentrations of $\mathrm{CO}_{2}, \mathrm{CH}_{4}$ and $\mathrm{CO}$ are analysed in Sect. 7.

The polarisation sensitivity of the geoCARB spectrometers imposes strong, wavelength dependent signatures upon the spectra, which raises the question as to whether such signatures might cause unacceptably large errors in retrieved concentrations of $\mathrm{CO}_{2}, \mathrm{CH}_{4}$ and $\mathrm{CO}$. Two experiments are conducted to assess this risk.

In the first, the inversion algorithm is denied access to the polarisation model of the instrument, thereby forcing it to assume that the measured signal represents the intensity at the top of the atmosphere. Although there is some degradation of accuracy for the retrieved concentrations of $\mathrm{CO}_{2}, \mathrm{CH}_{4}$ and $\mathrm{CO}$, the errors are not as large as might be expected, because the retrieval algorithm tries to attribute the wavelength signatures caused by the polarisation sensitivity of the gratings to the wavelength dependence of other geophysical parameters, especially the surface albedo. As the objective of the geoCARB mission is to measure trace gas concentrations, and not to measure albedos, the outcome of this experiment is marginally acceptable.

In the second experiment, the radiometric and polarimetric responses of geoCARB are assumed to be calibrated before launch, and the results are made available to the retrieval algorithm. In this case geoCARB returns trace gas concentrations with accuracy equal (on average) to that of a similar instrument equipped with polarisation scramblers. The latter ensure that the intensity reaching the detectors is the same (apart from a scaling factor) as the intensity arriving at the scan mirror. Thus, provided pre-flight calibration characterises both the radiometric and polarimetric responses of geoCARB, polarisation scramblers should not be needed. This is a fortunate result, because scramblers almost certainly would degrade the spatial resolution and increase both the instrument complexity and cost.

\section{Polarisation model}

The purpose of the polarisation model is to predict the signal at the detector ${ }^{2}$ from the Stokes vector ${ }^{3}$ of radiation arriving at the entrance aperture of geoCARB. Despite the complexity of the optical layout of geoCARB, shown in Fig. 1, in order to formulate the polarisation model it suffices to divide the optics of geoCARB into three logical assemblies, the first two being the moving scan mirrors (north-south and east-west), and the third being the fixed telescope and grating spectrograph. ${ }^{4}$ The division is shown schematically in Fig. 2, which also indicates the coordinate system used by geoCARB. All quantities in the polarisation model depend on wavelength, but the dependence is not shown explicitly in order to simplify the notation.

The transformation of the Stokes vector $S=(I, Q, U, V)^{\mathrm{T}}$ incident on the north-south scan mirror to the Stokes vector arriving at the detector is described by a Mueller matrix

$\mathbf{M}=\mathbf{M}_{3} \mathbf{M}_{2} \mathbf{M}_{1} \mathbf{R}_{\mathbf{0}}$.

The factor $\mathbf{R}_{0}$ rotates the plane of reference for polarisation from that used by the radiative transfer model to the reflection plane of the north-south scan mirror. It has the form

$\mathbf{R}_{0}=\mathbf{R}\left(\eta_{0}\right)$,

\footnotetext{
${ }^{2}$ The signal at the detector is represented by the output potential $v$, but equally well could be the output current or the charge accumulated over an integration period.

${ }^{3}$ The conventions for polarisation used by Mishchenko et al. (2002) are employed in this paper.

${ }^{4}$ In fact geoCARB contains two gratings, each used in two orders of diffraction, and four focal plane arrays. Where the text refers only to one spectrograph channel, similar arguments apply to all.
} 


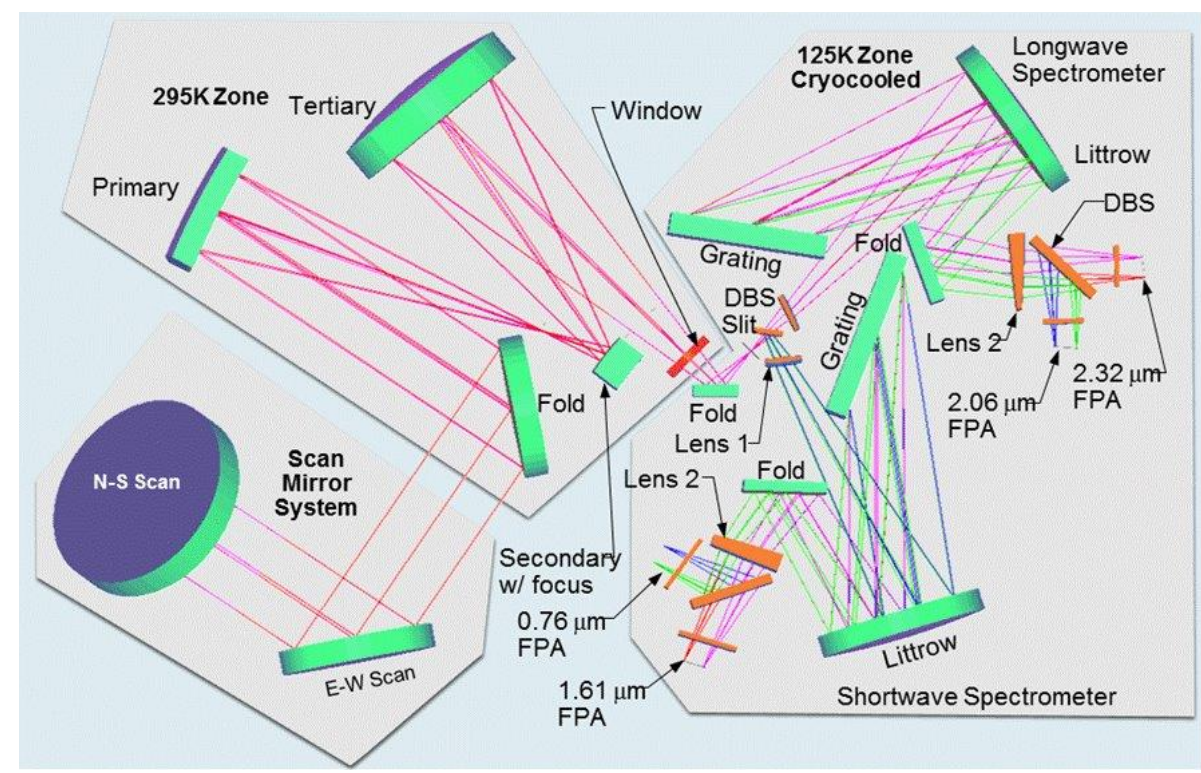

Figure 1. Optical layout for geoCARB. The primary beam splitter divides the long- and short-wave spectrometer arms. Each Littrow spectrometer feeds two separate focal plane arrays.

where $\eta_{0}$ is the angle between the two planes, and generally

$\mathbf{R}(\eta)=\left(\begin{array}{cccc}1 & 0 & 0 & 0 \\ 0 & +\cos 2 \eta & -\sin 2 \eta & 0 \\ 0 & +\sin 2 \eta & +\cos 2 \eta & 0 \\ 0 & 0 & 0 & 1\end{array}\right)$.

For the radiative transfer calculation, the reference plane for nadir viewing contains the ray from the sun to the target and the normal at the target. For non-nadir viewing, the normal and the ray from the target to the satellite are used. The rotation $\mathbf{R}_{0}$ is essentially a geometric quantity, and the degree of polarisation is preserved by the rotation.

The factor $\mathbf{M}_{1}$ represents the north-south scan mirror. It has the form

$\mathbf{M}_{\mathbf{1}}=\mathbf{R}\left(\eta_{1}\right) \mathbf{B}\left(\phi_{1}\right) \mathbf{A}\left(p_{1}, q_{1}\right)$

where

$\mathbf{A}(p, q)=\frac{1}{2}\left(\begin{array}{cccc}p^{2}+q^{2} & p^{2}-q^{2} & 0 & 0 \\ p^{2}-q^{2} & p^{2}+q^{2} & 0 & 0 \\ 0 & 0 & 2 p q & 0 \\ 0 & 0 & 0 & 2 p q\end{array}\right)$

accounts for Fresnel reflection at the mirror surface with

$p^{2}=r_{\|} \quad$ and $\quad q^{2}=r_{\perp}$,

and $r_{\|}$and $r_{\perp}$ are the reflection coefficients for linearly polarised light parallel and perpendicular to the plane of reflection. The factor $\mathbf{B}\left(\phi_{1}\right)$ accounts for phase shift caused (principally) by the optical coating of the mirror. The matrix $\mathbf{B}$ has the general form

$\mathbf{B}(\phi)=\left(\begin{array}{cccc}1 & 0 & 0 & 0 \\ 0 & 1 & 0 & 0 \\ 0 & 0 & \cos \phi & \sin \phi \\ 0 & 0 & -\sin \phi & \cos \phi\end{array}\right)$,

where the angle $\phi$ is the advancement of the phase of light linearly polarised parallel to the reflection plane relative to light linearly polarised perpendicular to the reflection plane. Because the matrices $\mathbf{A}(p, q)$ and $\mathbf{B}(\phi)$ commute, the order in which they are written is immaterial. The final factor $\mathbf{R}\left(\eta_{1}\right)$ accounts for the rotation through angle $\eta_{1}$ between the reflection planes of the north-south and east-west scan mirrors. The reflection coefficients $r_{\|}$and $r_{\perp}$ and the phase shift $\phi$ are functions of wavelength and the angle of incidence, which must be characterised during radiometric and polarimetric calibration.

The factor $\mathbf{M}_{2}$ also has the form

$\mathbf{M}_{2}=\mathbf{R}\left(\eta_{2}\right) \mathbf{B}\left(\phi_{2}\right) \mathbf{A}\left(p_{2}, q_{2}\right)$,

where now $p_{2}, q_{2}$ and $\phi_{2}$ refer to properties of the east-west scan mirror. The angle $\eta_{2}$ appearing in the rotation $\mathbf{R}\left(\eta_{2}\right)$ is the angle between the reflection plane of the east-west scan mirror and the reference plane for the spectrograph. The latter is defined by the optic axis and the projection of the long axis through the spectrograph slit onto the east-west scan mirror.

Finally, the factor $\mathbf{M}_{3}$ in Eq. (1) describes the telescope and grating spectrograph assembly. Despite the optical complexity of the system, as indicated in Fig. 1, because it is fixed 
it may be represented by a single matrix,

$\mathbf{M}_{3}=\left(\begin{array}{llll}m_{00} & m_{01} & m_{02} & m_{03} \\ m_{10} & m_{11} & m_{12} & m_{13} \\ m_{20} & m_{21} & m_{22} & m_{23} \\ m_{30} & m_{31} & m_{32} & m_{33}\end{array}\right)$,

whose elements are to be determined via calibration.

Let $S_{0}$ denote the Stokes vector incident on the northsouth scan mirror, as computed by the radiative transfer model. Let

$S_{1}=\mathbf{M}_{1} \mathbf{R}_{0} S_{0}, \quad S_{2}=\mathbf{M}_{2} S_{1}$ and $S_{3}=\mathbf{M}_{3} S_{2}$

similarly denote the Stokes vectors immediately before the east-west scan mirror, the telescope/spectrograph assembly and the detector. During pre-flight calibration of geoCARB, the reflection coefficients and phase shifts, $p_{i}, q_{i}$ and $\phi_{i}$, associated with the scan mirrors will be determined as functions of wavelength and angle of incidence, so the matrices $\mathbf{A}$ and $\mathbf{B}$ will be known. Furthermore, because the geometry of observation will be known, so too will the angles $\eta_{0}, \eta_{1}$ and $\eta_{2}$ appearing in the rotation matrices. Thus, the Mueller matrices $\mathbf{R}_{0}, \mathbf{M}_{1}$ and $\mathbf{M}_{2}$ associated with the scan mirrors, and hence the Stokes vectors $\boldsymbol{S}_{1}$ and $\boldsymbol{S}_{2}$, can be calculated.

We assume that the detector responds only to the intensity incident upon its surface. Because

$I_{3}=m_{00} I_{2}+m_{01} Q_{2}+m_{02} U_{2}+m_{03} V_{2}$,

where $I_{2}, Q_{2}, U_{2}$ and $V_{2}$ may be considered known, only the elements $m_{00}, m_{01}, m_{02}$ and $m_{03}$ of the first row of the Mueller matrix for the telescope/spectrograph assembly must be determined by the pre-flight polarimetric calibration. How this will be done is outlined in the next section.

The output potential $v$ from the detector is assumed to be a (mildly) non-linear function of the intensity incident upon the detector,

$v=g\left(I_{3}\right)$.

For example the function $g$ might be a polynomial in the intensity, such as

$v=g_{0}+g_{1} I_{3}+g_{2} I_{3}^{2}$,

where the coefficients $g_{0}, g_{1}$ and $g_{2}$ are to be determined during the pre-flight radiometric calibration.

In summary, the polarisation model requires the following:

1. geometric calculations to provide the rotation angles $\eta_{0}$, $\eta_{1}$ and $\eta_{2}$

2. optical properties of the scan mirrors;

3. elements $m_{00}, m_{01}, m_{02}$ and $m_{03}$ of the Mueller matrix for the telescope/spectrograph assembly;

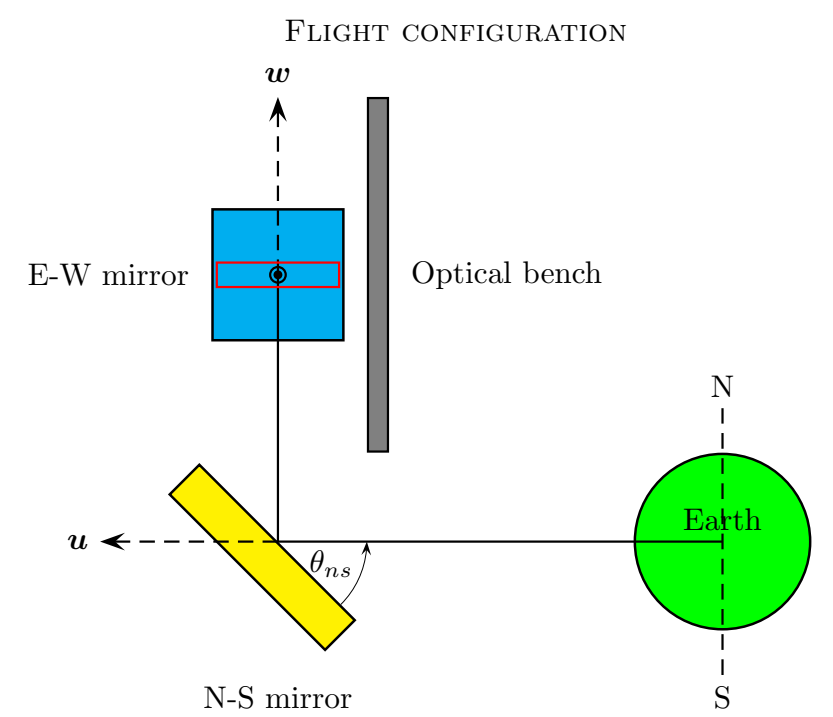

Figure 2. The schematic shows the coordinate system and orthogonal unit vectors $\boldsymbol{u}, \boldsymbol{v}$ and $\boldsymbol{w}$ used for geoCARB. The nadir direction from the centre of the north-south scan mirror to the centre of the earth defines the negative $\boldsymbol{u}$ axis. The positive $\boldsymbol{v}$ axis points eastward along the equator. In the schematic, it is represented by the arrowhead emerging from the page in the centre of the east-west scan mirror. The $\boldsymbol{w}$ axis, defined by $\boldsymbol{w}=\boldsymbol{u} \times \boldsymbol{v}$, points to the north. The optical bench is parallel to the satellite platform, and its normal vector is parallel to $\boldsymbol{u}$. The image of the slit on the east-west scan mirror is indicated by the red rectangle. The slit also is parallel to $\boldsymbol{u}$. The north-south scan mirror rotates about the $\boldsymbol{v}$ axis through the angle denoted $\theta_{\mathrm{ns}}$ in the schematic. The east-west scan mirror rotates about the $\boldsymbol{u}$ axis through angle $\theta_{\text {ew }}$ (not shown).

4. parameters (such as $g_{0}, g_{1}$ and $g_{2}$ ) that characterise the response of the detector to the intensity incident upon it.

Once these quantities have been specified, the calculation reduces to a simple matrix transformation of the Stokes vector incident upon the north-south scan mirror.

\section{Radiometric and polarimetric calibration}

During radiometric and polarimetric calibration, the northsouth and east-west scan mirrors will be set at their central positions $\left(\theta_{\mathrm{ns}}=\pi / 4\right.$ and $\left.\theta_{\mathrm{ew}}=\pi / 4\right)$ so that the instrument points to nadir along the negative $\boldsymbol{u}$ axis, as shown schematically in Fig. 3. Unpolarised light from a well-calibrated integrating sphere will be directed along the optic axis onto the scan mirror through a linear polariser that can be rotated about the optic axis through angle $\theta$. For the calibration configuration, the plane used to define the incident Stokes vector is the $u-w$ plane, which also is the plane of reflection for the north-south mirror. 
The Stokes vector after reflection from the north-south mirror will be

$\boldsymbol{S}_{1}=\mathbf{R}\left(\eta_{1}\right) \mathbf{B}\left(\phi_{1}\right) \mathbf{A}\left(p_{1}, q_{1}\right) \mathbf{L}(\theta) \boldsymbol{S}_{0}$,

where $\boldsymbol{S}_{0}=\left(I_{0}, 0,0,0\right)^{\mathrm{T}}$ is the Stokes vector for unpolarised light leaving the integrating sphere,

$\mathbf{L}(\theta)=\frac{1}{2}\left(\begin{array}{cccc}1 & c & s & 0 \\ c & c^{2} & c s & 0 \\ s & s c & s^{2} & 0 \\ 0 & 0 & 0 & 0\end{array}\right)$

is the Mueller matrix for the linear polariser inclined at angle $\theta$, and

$c=\cos 2 \theta$ and $s=\sin 2 \theta$.

The plane containing the incident and reflected beams at the east-west mirror is the $v-w$ plane, perpendicular to the corresponding plane for the north-south mirror. Therefore, $\eta_{1}=\pi / 2$ and

$\mathbf{R}\left(\eta_{1}\right)=\left(\begin{array}{rrrr}1 & 0 & 0 & 0 \\ 0 & -1 & 0 & 0 \\ 0 & 0 & -1 & 0 \\ 0 & 0 & 0 & 1\end{array}\right)$.

A straightforward calculation yields

$\boldsymbol{S}_{1}=\frac{I_{0}}{4}\left(\begin{array}{c}p_{1}^{2}(1+c)+q_{1}^{2}(1-c) \\ -p_{1}^{2}(1+c)+q_{1}^{2}(1-c) \\ -2 p_{1} q_{1} \cos \phi_{1} s \\ -2 p_{1} q_{1} \sin \phi_{1} s\end{array}\right)$.

The Stokes vector leaving the east-west mirror and arriving at the entrance aperture of the telescope is

$\boldsymbol{S}_{\mathbf{2}}=\mathbf{R}\left(\eta_{2}\right) \mathbf{B}\left(\phi_{2}\right) \mathbf{A}\left(p_{2}, q_{2}\right) \boldsymbol{S}_{1}$.

In the calibration configuration no rotation occurs between the east-west scan mirror and the telescope/spectrograph assembly, so $\eta_{2}=0$ and the matrix $\mathbf{R}\left(\eta_{2}\right)$ is the identity. Thus, Eq. (19) reduces to

$\boldsymbol{S}_{2}=\frac{I_{0}}{4}\left(\begin{array}{c}p_{2}^{2} q_{1}^{2}(1-c)+q_{2}^{2} p_{1}^{2}(1+c) \\ p_{2}^{2} q_{1}^{2}(1-c)-q_{2}^{2} p_{1}^{2}(1+c) \\ -2 p_{1} q_{1} p_{2} q_{2} \cos \left(\phi_{1}-\phi_{2}\right) s \\ -2 p_{1} q_{1} p_{2} q_{2} \sin \left(\phi_{1}-\phi_{2}\right) s\end{array}\right)$,

while the intensity component of the Stokes vector incident upon the detector will be

$$
\begin{aligned}
I_{3}=\frac{I_{0}}{4}[ & m_{00}\left[p_{2}^{2} q_{1}^{2}(1-c)+q_{2}^{2} p_{1}^{2}(1+c)\right] \\
& +m_{01}\left[p_{2}^{2} q_{1}^{2}(1-c)-q_{2}^{2} p_{1}^{2}(1+c)\right] \\
& -2 m_{02} p_{1} q_{1} p_{2} q_{2} \cos \left(\phi_{1}-\phi_{2}\right) s
\end{aligned}
$$

$$
\left.-2 m_{03} p_{1} q_{1} p_{2} q_{2} \sin \left(\phi_{1}-\phi_{2}\right) s\right]
$$

with corresponding output potential $v$ from the detector

$v=g\left(I_{3}\right)$

In practice the linear polariser will be set at angles

$0=\theta_{1}<\theta_{2}<\cdots<\theta_{n}=\pi / 2$

and for each angle the output potential will be measured as the incident intensity $I_{0}$ is stepped over the range likely to be encountered by geoCARB in space,

$I_{01}<I_{02}<\cdots<I_{0 k}$.

Thus, for angle $\theta_{i}$ and incident intensity $I_{0 j}$, there will be a corresponding output potential

$v_{i j}=g\left(I_{0 j}, \theta_{i}, m_{00}, m_{01}, m_{02}, m_{03}\right)$.

Provided that the north-south and east-west scan mirrors have been characterised well, the $n k$ measurements of $v_{i j}$ will provide an over-determined system of equations for the elements $m_{00}, m_{01}, m_{02}$ and $m_{03}$ of the Mueller matrix as well as the parameters (such as $g_{0}, g_{1}$ and $g_{2}$ ) that define the function $g$. Solution of the over-determined system in a leastsquares sense will characterise both the polarimetric and radiometric sensitivity of the spectrograph from the entrance aperture of the telescope through to the output from the detector.

It is important to note the role played by the phase delays $\phi_{1}$ and $\phi_{2}$ in Eq. (21). If $\phi_{1} \approx \phi_{2}$, as is likely to be the case with similar coatings on the mirrors, then $m_{03}$ will be difficult to determine because its coefficient in Eq. (21) will be close to zero. That might not be a serious problem in practice, because the surface and atmosphere generate very little circular polarisation. However, if necessary, a well-characterised retarder could be introduced to the calibration set-up between the integrating sphere and the linear polariser to ensure a significant component of circular polarisation, thereby leading to a more accurate determination of $m_{03}$. These matters will be addressed during the phase A study for geoCARB.

Once geoCARB is in flight, the stability of the polarimetric calibration will be monitored using observations of sunglint in a manner similar to that devised for GOSAT by O'Brien et al. (2013).

\section{Simplified configuration}

In order to assess the polarisation sensitivity of geoCARB with information presently available, we consider a simplified (and idealised) configuration ${ }^{5}$ in which

\footnotetext{
${ }^{5}$ This simplified instrument is likely to be more polarising than geoCARB.
} 
- the mirrors are perfectly reflecting, so that $p_{i}=q_{i}=1$, and the phase delays $\phi_{1}$ and $\phi_{2}$ are equal;

- the polarising properties of the telescope/spectrograph assembly are dominated by the grating;

- the intensity reflected from the grating when illuminated with plane-polarised light inclined at angle $\theta=\pi / 4$ to the rulings is the average of the intensities at $\theta=0$ and $\theta=\pi / 2$.

In practice, the last assumption requires that incident radiation linearly polarised parallel to the grating rulings should not produce any diffracted light linearly polarised perpendicular to the rulings, and vice-versa. With these assumptions, Eq. (21) for the intensity arriving at the detector during calibration with the polariser at angle $\theta$ reduces to

$I=\frac{I_{0}}{2}\left(m_{00}-m_{01} \cos 2 \theta-m_{02} \sin 2 \theta\right)$,

where for notational simplicity we have omitted the subscript from $I_{3}$.

\subsection{Polarimetric calibration}

If we assume that the atmosphere generates little circular polarisation, then only three parameters are required to characterise the instrument, namely $m_{00}, m_{01}$ and $m_{02}$. In principle only three measurements are needed to fix their values, which for definiteness we assume to be the responses $I^{(1)}, I^{(2)}$ and $I^{(3)}$ to unpolarised intensity $I_{0}$ with the linear polariser at angles $0, \pi / 4$ and $\pi / 2$. Substitution of these angles in Eq. (26) leads to

$I^{(1)}=I_{0}\left(m_{00}-m_{01}\right) / 2$,

$I^{(2)}=I_{0}\left(m_{00}-m_{02}\right) / 2$,

$I^{(3)}=I_{0}\left(m_{00}+m_{01}\right) / 2$.

The first and third equations yield

$m_{00}-m_{01}=E_{p}$ and $m_{00}+m_{01}=E_{s}$,

where the ratios

$E_{p}=2 I^{(1)} / I_{0}$ and $E_{s}=2 I^{(3)} / I_{0}$

are the absolute efficiencies of the grating for linearly polarised light parallel and perpendicular to the rulings. Thus, we obtain

$m_{00}=\left(E_{s}+E_{p}\right) / 2$ and $m_{01}=\left(E_{s}-E_{p}\right) / 2$,

showing that the coefficients $m_{00}$ and $m_{01}$ can be expressed simply in terms of the grating efficiencies measured by the manufacturer. Figure 4 shows the absolute efficiencies of the geoCARB gratings, measured by the manufacturer in the $\mathrm{O}_{2}$

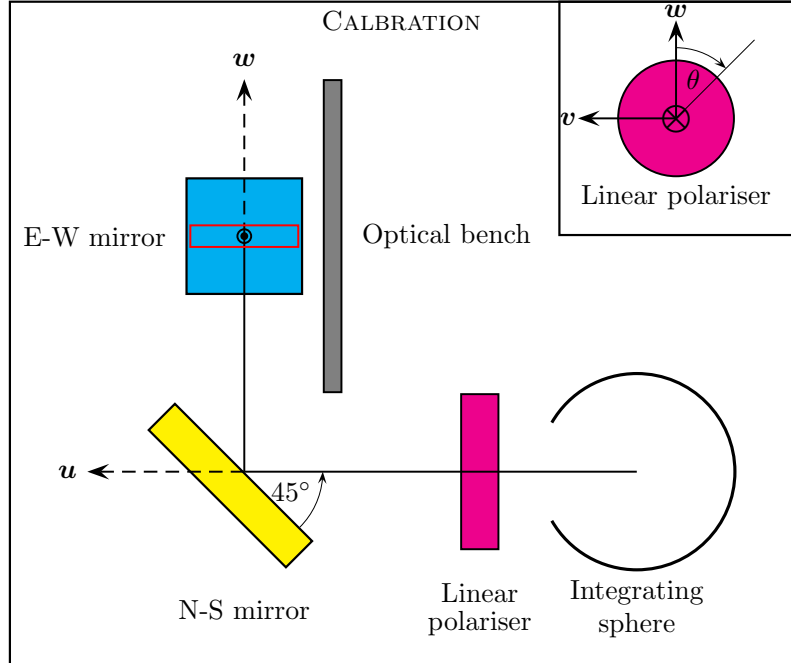

Figure 3. During calibration, both mirrors will be set to their central positions with $\theta_{\mathrm{ns}}=\theta_{\mathrm{ew}}=\pi / 4$, corresponding to nadir observation. Unpolarised light from a calibrated integrating sphere will be passed through a linear polariser along the optic axis to the northsouth scan mirror. The polariser will be rotated about the $\boldsymbol{u}$ axis so that the plane of polarisation makes an angle $\theta$ with the $u-w$ plane, as shown in the upper-right insert. When $\theta=0$, the plane of polarisation (after reflections) is parallel to the slit; when $\theta=\pi / 2$, the plane of polarisation is perpendicular to the slit. The output potential $v(\theta)$ from the detector will be monitored as a function of $\theta$.

A-band and the weak $\mathrm{CO}_{2}$ band, and predicted in the strong $\mathrm{CO}_{2}$ band and the $\mathrm{CO}$ band.

The last assumption concerns the sensitivity of the grating to the $U$ component of the radiation incident upon it. The second of the relations in Eq. (27) shows that

$I^{(2)}=\left(I^{(1)}+I^{(3)}\right) / 2-m_{02} I_{0} / 2$.

Therefore, the requirement that $I^{(2)}$ should be the average of $I^{(1)}$ and $I^{(3)}$ forces $m_{02}=0$, which completes the characterisation of the simplified spectrograph. Without this requirement, $m_{02}$ could be determined from the measurement $I^{(2)}$.

\subsection{In-flight operation}

Once in flight, the intensity falling upon the detector of the simplified instrument in response to the Stokes vector $S=$ $(I, Q, U, V)^{\mathrm{T}}$ at the top of the atmosphere will be simply

$I_{3}=m_{00} I_{0}+m_{01} Q_{0}$,

where

$I_{0}=I \quad$ and $\quad Q_{0}=\cos 2 \eta_{0} Q-\sin 2 \eta_{0} U$.

The Stokes component $U_{0}$ does not appear in Eq. (32) because $m_{00}$ and $m_{01}$ are the only non-zero Stokes coefficients. 
The angle between the reference planes used by the radiative transfer code and the instrument is $\eta_{0}$; it is a purely geometric quantity that depends upon the orbit and the scan geometry. For example, Fig. 5 shows the angle $\eta_{0}$ for pixels in the frames through Agra, Wuhan and Alice Springs. The variation in $\eta_{0}$ is small when the target is close to the longitude of geoCARB, but elsewhere can be large. If we define

$H(\lambda)=\frac{2 E_{s}}{E_{s}+E_{p}} \quad$ and $\quad V(\lambda)=\frac{2 E_{p}}{E_{s}+E_{p}}$,

then Eq. (32) reduces to

$I_{3}=m_{00}\left[I_{0}+(H-V) Q_{0} / 2\right]$.

Thus, the intensity reaching the detector for this idealised instrument is identical to that generated by unpolarised intensity

$I^{\star}=I_{0}+(H-V) Q_{0} / 2$

incident upon the north-south scan mirror.

\section{Pseudo measured spectra}

An ensemble of spectra were generated for targets in frames passing through Agra, Wuhan and Alice Springs, as described in Sect. 1. Only land targets were selected for this study because generally the oceans are too dark at the geoCARB wavelengths.

The meteorology at each target was based on forecasts from the European Centre for Medium-Range Weather Forecasts (ECMWF), interpolated to the time and location of each observation. ${ }^{6}$ Surface properties were derived from MODIS and POLDER, which respectively provided the bidirectional reflectance distribution function and polarising properties (Nadal and Breon, 1999). Clouds and aerosols were derived from CALIPSO (Cloud-Aerosol Lidar and Infrared Pathfinder Satellite Observations). The vertical profiles of $\mathrm{CO}_{2}$ in the simulator were derived from the Parameterised Chemical Transport Model (PCTM) (Kawa et al., 2004). For $\mathrm{CO}$, the background profiles were drawn from the Measurements of Pollution in the Troposphere (MOPITT) mission (Deeter et al., 2003, 2007a, b). Profiles of $\mathrm{CH}_{4}$ were taken from a snap-shot of the global $\mathrm{CH}_{4}$ distribution calculated with the TM5 chemical transport model (Krol et al., 2005). In each case, the profiles were interpolated to the times and locations of the geoCARB observations. Generally the methods were identical to those described by Polonsky et al.

\footnotetext{
${ }^{6}$ The specific dates for the simulations were the 21 st of March, June, September and December in 2012. The equinoxes and solstices were chosen to capture the seasonal dependence. The only significance of the year 2012 is that data were already on hand for the geophysical variables; we expect similar results for other years. Three observations were simulated for each day, at local solar noon, 3 hours earlier and 3 hours later.
}

(2014), except that superimposed on the column concentrations of $\mathrm{CO}_{2}, \mathrm{CH}_{4}$ and $\mathrm{CO}$ were random variations drawn from gaussian distributions with standard deviations of 3.0, 0.1 and $0.01 \mathrm{ppm}$, respectively. The random variations were added simply to augment the parameter space sampled by the simulations. Similarly, the simulations were performed twice, once with both cloud and aerosol enabled and once with only aerosol, the aim being to generate a larger ensemble of "almost clear" scenes with which to test the sensitivity of the retrieval algorithm to polarisation. This approach is reasonable because moderately cloudy scenes are rejected by the algorithm.

Histograms of surface pressure and the column-averaged concentrations of $\mathrm{CO}_{2}, \mathrm{CH}_{4}$ and $\mathrm{CO}$ are shown in Fig. 7 for the ensemble of pixels in the frames over Agra, Wuhan and Alice Springs. Figure 8 presents histograms of the optical depth at the $\mathrm{O}_{2}$ A-band of cloud liquid water, cloud ice and aerosol. The histograms in blue represent the entire ensemble; those in red show the ensemble members that pass the post-processing filter.

For each target, all components of the Stokes vector $\boldsymbol{S}=$ $(I, Q, U, V)^{\mathrm{T}}$ were computed at the top of the atmosphere, with the reference plane for polarisation defined by the local normal and the direction to the satellite at the target. The spectral channels, their widths and the signal-to-noise ratios were as described for geoCARB by Polonsky et al. (2014). In particular, the instrument line shape functions were assumed to be independent of polarisation. The polarisation model for the idealised instrument was applied to the Stokes vector to calculate the intensity falling upon the detector. As shown earlier, the response of the detector is identical to that produced by unpolarised light at the entrance aperture with intensity given by Eq. (34). Because $H$ and $V$ depend strongly upon wavelength, the measured spectrum contains an artefact arising from the polarisation sensitivity of the gratings.

The Stokes vector $\boldsymbol{S}$ was computed using a three-step approach: calculate the exact contribution to $S$ from first-order scattering (1OS); calculate the multiply scattered radiance $I$ at the top of the atmosphere $\left(I_{\mathrm{ms}}\right)$; calculate the contributions from second-order scattering to $Q$ and $U$, as well as the polarisation corrections from second-order scattering to $I$ (2OS). By combining the results of these calculations, the Stokes vector at the top of the atmosphere can be estimated reasonably accurately for nearly clear scenes (Natraj and Spurr, 2007). The 1 OS and 2OS terms used code developed by Natraj and Spurr (2007). Calculation of the first-order component of $I$ used the TMS (truncated multiple scattering) correction of Nakajima and Tanaka (1988), and all three first-order scattering terms include the direct beam scattered from the surface. The multiply scattered intensity term $I_{\mathrm{ms}}$ is calculated using the successive orders of interaction (SOI) radiative transfer model (Heidinger et al., 2006) with slight updates for the infrared. The SOI model employs the delta-M phase function truncation technique of Wiscombe (1977). The SOI model is both fast and accurate 

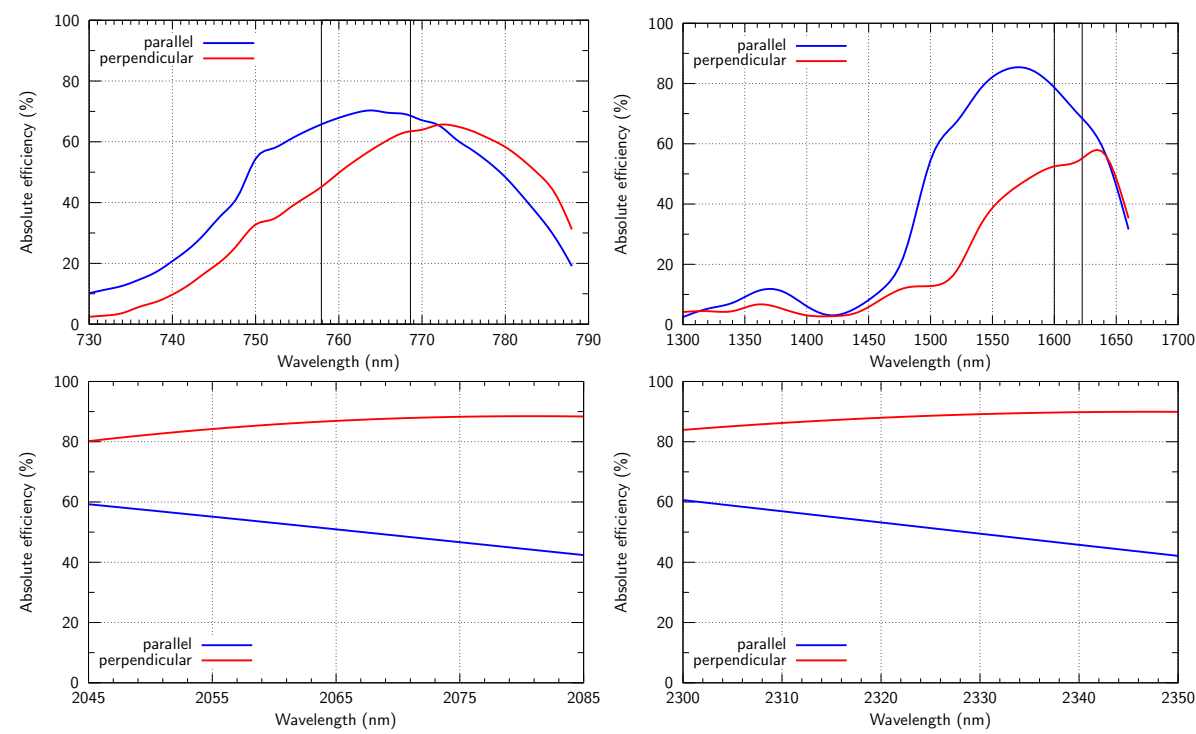

Figure 4. Absolute efficiencies of the gratings, measured in the $\mathrm{O}_{2} \mathrm{~A}$-band and weak $\mathrm{CO}_{2}$ band, and predicted in the strong $\mathrm{CO}_{2}$ band and $\mathrm{CO}$ band. There are two gratings, each used in two orders of diffraction to serve two bands. The vertical lines define the $\mathrm{O}_{2} \mathrm{~A}-\mathrm{band}$ and $\mathrm{CO}_{2}$ weak band.
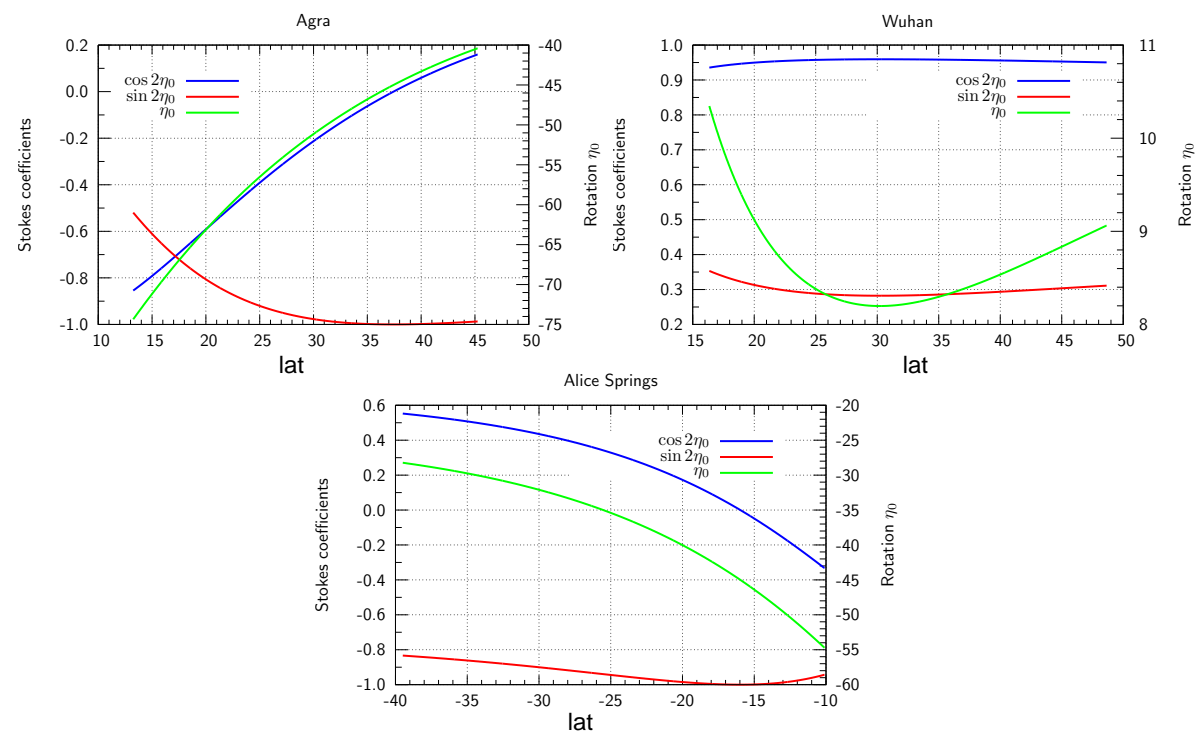

Figure 5. Angle $\eta_{0}$ (right-hand scale) between the reference planes for polarisation used by the radiative transfer code and the geoCARB instrument, shown as a function of latitude along the frames through Agra, Wuhan and Alice Springs. Also plotted are cos $2 \eta_{0}$ and sin $2 \eta_{0}$ (left-hand scale), which are essentially the Stokes coefficients for the simplified model of the instrument (hence the left-hand label).

(O’Dell et al., 2006). Lastly, the techniques of low-streams interpolation (LSI) developed by O'Dell (2010) was used to compute the Stokes vector on a $0.01 \mathrm{~cm}^{-1}$ spectral grid; high accuracy, but widely spaced, radiances were interpolated to the fine spectral grid using a two-stream solver of the radiative transfer equation.

Generally, in simulations of this type, random noise would be added to the unpolarised intensity in accordance with the noise model for geoCARB, and the resulting signal would be regarded as a measurement (or measured spectrum).

However, in this study random noise was not added for the following reason. For every retrieval, differences between the true and retrieved values of the parameters can arise via many mechanisms, including the following:

1. differences between the absorption coefficients and radiative transfer models used for the forward simulation and for the retrieval algorithm; 

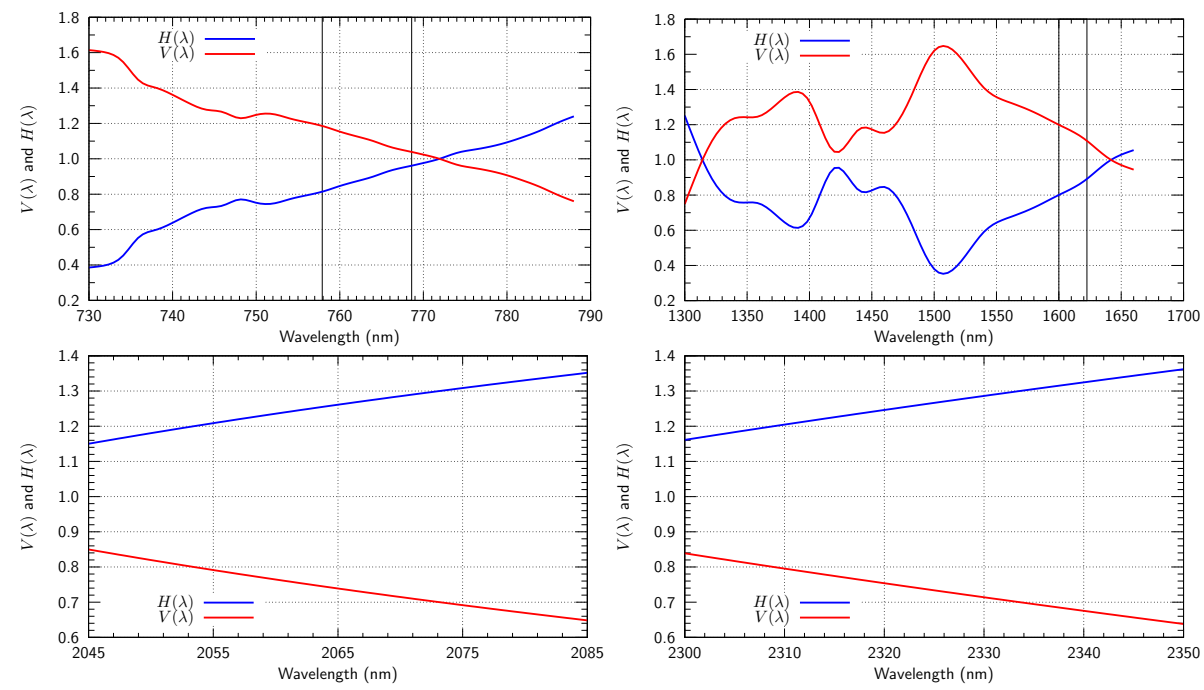

Figure 6. Functions $V(\lambda)$ and $H(\lambda)$ for the gratings, measured in the $\mathrm{O}_{2}$ A-band and weak $\mathrm{CO}_{2}$ band, and predicted in the strong $\mathrm{CO}_{2}$ band and $\mathrm{CO}$ band. There are two gratings, each used in two orders of diffraction to serve two bands. The vertical lines define the $\mathrm{O}_{2} \mathrm{~A}-\mathrm{band}$ and $\mathrm{CO}_{2}$ weak band.

Table 1. Coefficients in the linear approximations to $H(\lambda)$ and $V(\lambda)$. Wavelength $\lambda$ is assumed in $\mathrm{nm}$.

\begin{tabular}{lrr}
\hline Band & $\alpha\left(\mathrm{nm}^{-1}\right)$ & $\beta$ \\
\hline $\mathrm{O}_{2}$ A-band & 0.01439 & -10.825 \\
Weak $\mathrm{CO}_{2}$ band & 0.00389 & -6.426 \\
Strong $\mathrm{CO}_{2}$ band & 0.00501 & -10.095 \\
$\mathrm{CO}$ band & 0.00404 & -9.118 \\
\hline
\end{tabular}

2. the influence of the prior and algorithm controls, such as the stopping condition;

3. random noise added to the simulated spectra.

The last source is the most understood, and its magnitude can be quantified easily by the posterior uncertainties returned by the retrieval algorithm, the calculation of which uses the instrument signal-to-noise ratio. Furthermore, random noise in the spectra generally will not cause a bias, because the radiative transfer problem can be linearised in the vicinity of the true solution. Consequently, we can concentrate on the biases introduced by factors other than random noise (such as the first two items listed above). Since the model errors and the random noise (items 1 and 3) are statistically independent, including the effects of random noise simply widens the bias distribution by the width of the random uncertainty. As the focus of this study is the bias introduced by polarisation effects, it was judged that the effects would be easier to spot in the narrower error distributions calculated without random noise.

\section{Trace gas recovery}

Optimal estimation was used to match "measured" (in reality simulated) and modelled spectra, as described by Polonsky et al. (2014) for the baseline configuration of geoCARB. In addition to the trace gas $\left(\mathrm{CO}_{2}, \mathrm{CH}_{4}\right.$ and $\left.\mathrm{CO}\right)$ concentrations, the state vector contained many other parameters describing the surface, the atmosphere and the scattering properties of aerosol and cloud. All were adjusted iteratively during the matching process.

In contrast to the measured spectra, which were computed using polarising surfaces with directional reflectance, the modelled spectra assumed that the surfaces were nonpolarising and Lambertian, with albedo varying linearly with wavelength. An estimate for the albedo was derived from the spectra using a selection of frequencies, mostly in the continuum, and a radiometric model that assumed the atmosphere was free of cloud and aerosol. The estimate so obtained then was used as both the first guess and the prior in Rogers' optimal estimation. Thus, while the modelled surface was based on reasonable prior information, it differed in detail from the measured surface. This difference ensured that simulation followed by retrieval was not a circular process, and in fact was open to the range of errors we expect with real data.

Similarly, the measured spectra used cloud and aerosol profiles observed by CALIPSO, whereas the modelled spectra assumed two types of aerosol plus liquid water and ice clouds with effective radii of 8 and $70 \mu \mathrm{m}$ respectively. The vertical profiles of particulates were assumed to be gaussian in shape. The optical thicknesses of aerosol, cloud liquid water and cloud ice, in addition to the heights and widths of the vertical distributions, were adjusted when fitting modelled to measured spectra. Thus, the modelled aerosol and 
Table 2. Means $(\mu)$ and standard deviations $(\sigma)$ of the biases $\delta X_{\mathrm{CO}_{2}}, \delta X_{\mathrm{CH}_{4}}, \delta X_{\mathrm{CO}}$ and $\delta p_{s}$ in retrieved $X_{\mathrm{CO}_{2}}, X_{\mathrm{CH}_{4}}, X_{\mathrm{CO}}$ and surface pressure from the two experiments. The row labelled "unpolarised" contains reference results obtained for an instrument equipped with ideal polarisation scramblers.

\begin{tabular}{ccccccccc}
\hline Experiment & \multicolumn{2}{c}{$\delta X_{\mathrm{CO}_{2}}(\mathrm{ppm})$} & \multicolumn{2}{c}{$\delta X_{\mathrm{CH}_{4}}(\mathrm{ppb})$} & \multicolumn{2}{c}{$\delta X_{\mathrm{CO}}(\mathrm{ppb})$} & \multicolumn{2}{c}{$\delta p_{s}(\mathrm{hPa})$} \\
& $\mu$ & $\sigma$ & $\mu$ & $\sigma$ & $\mu$ & $\sigma$ & $\mu$ & $\sigma$ \\
\hline 1 & -0.79 & 1.40 & 5.88 & 8.35 & -3.51 & 13.33 & -0.06 & 2.39 \\
2 & -0.63 & 0.98 & 6.54 & 6.93 & -3.23 & 13.01 & -0.37 & 1.89 \\
unpolarised & -0.67 & 1.09 & 6.32 & 7.09 & -3.75 & 13.22 & -0.30 & 1.97 \\
\hline
\end{tabular}
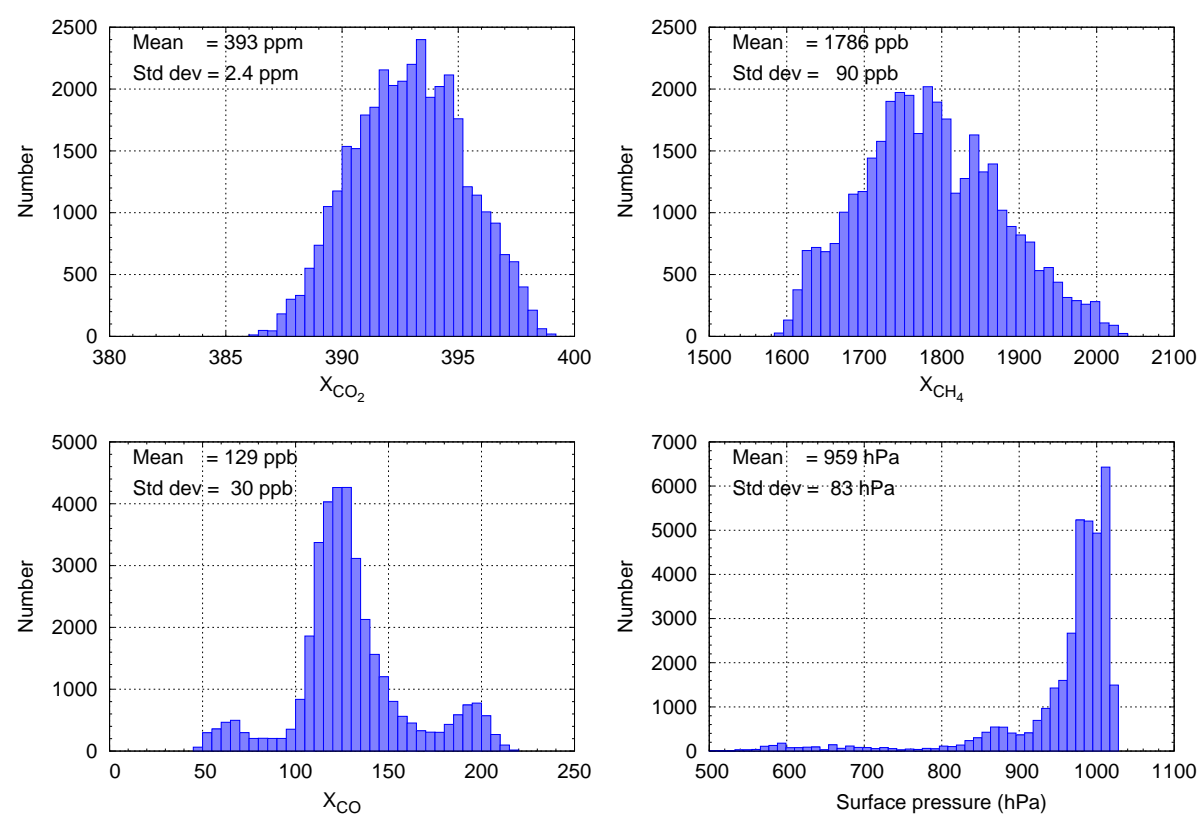

Figure 7. Histograms of $X_{\mathrm{CO}_{2}}, X_{\mathrm{CH}_{4}}, X_{\mathrm{CO}}$ and surface pressure for the ensemble of soundings in the simulation. The surface pressure histogram covers a wide range because the frame passing through Agra includes the Himalaya.

cloud could differ significantly from the aerosol and cloud from CALIPSO used in the simulation of the measured spectra, again breaking the circularity of the simulation-retrieval process.

For each day, each observation time and each (approximately) north-south scan line (through Agra, Wuhan or Alice Springs), the prior profile of $\mathrm{CO}_{2}$ was taken to be the average of the profiles at all of the target pixels along the scan line. This was judged to be a fair prior, neither too optimistic nor too pessimistic, and indicative of the accuracy possible with large-scale averages predicted by general circulation models. Prior profiles of $\mathrm{CH}_{4}$ and $\mathrm{CO}$ were calculated similarly.

At the completion of the optimal estimation, a postprocessing filter (PPF) is applied to reject cases where the model approximation to the spectra is poor. This may happen for many reasons, but the majority of cases occur when the optical properties assumed for aerosol and cloud do not match those used to simulate the spectra. The experiments in this study used the same PPF as Polonsky et al. (2014). The PPF checks $\chi^{2}$ in the bands used to retrieve $X_{\mathrm{CO}_{2}}$, the retrieved aerosol optical depth at the blue end of the $\mathrm{O}_{2}$ Aband and the number of degrees of freedom for signal in the retrieved profile of $\mathrm{CO}_{2}$. Each check involves comparison with a fixed, preset threshold. If any check fails, the scene is rejected. Only results that pass the PPF are shown.

The functions $H(\lambda)$ and $V(\lambda)$ derived from the efficiencies of the gratings to polarisations parallel and perpendicular to the slits were approximated by linear functions, which take the form

$H=\alpha \lambda+\beta+1 \quad$ and $\quad V=-\alpha \lambda-\beta+1$

because $H+V=2$ by definition. The coefficients $\alpha$ and $\beta$ are listed in Table 1. Quadratic approximations produce almost identical results. Over all bands, $H$ and $V$ vary by approximately $15 \%$, so their dependence on wavelength is strong, but slow in comparison with the rate at which the gas absorption spectrum varies. 

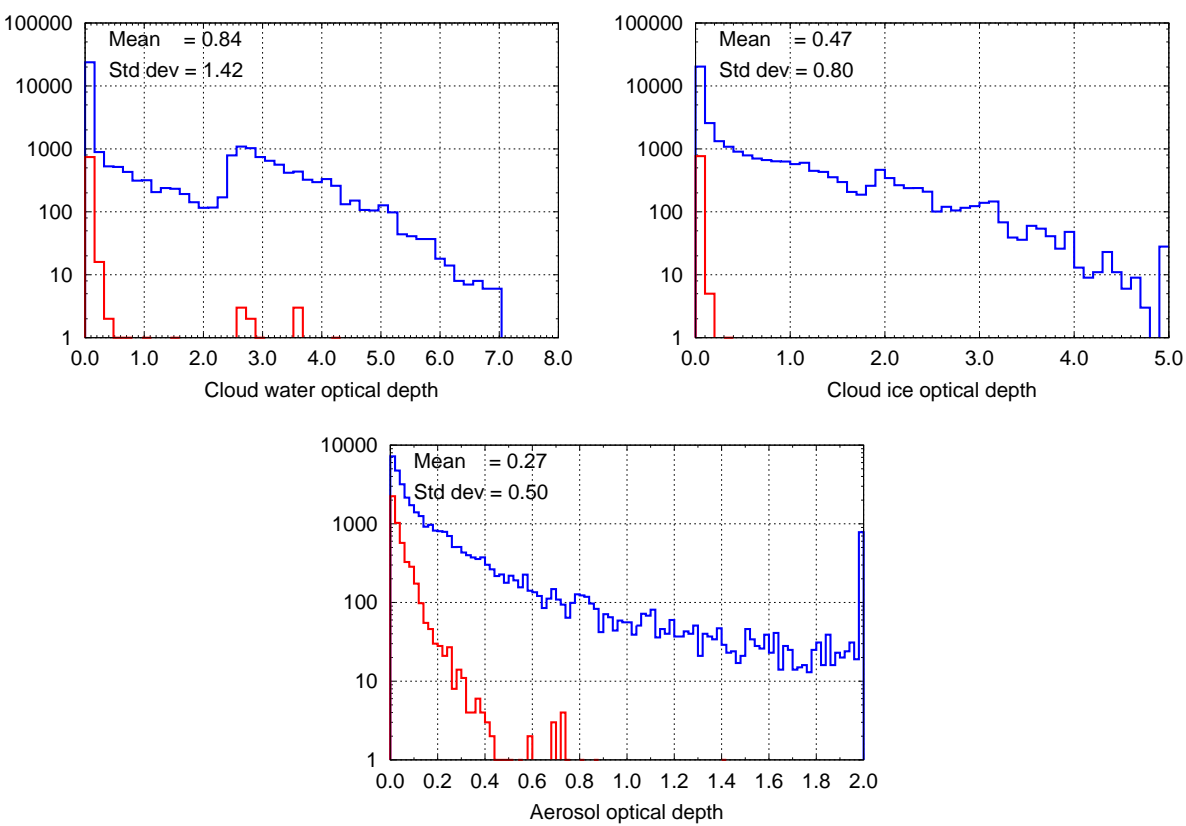

Figure 8. Histograms of the optical depth of cloud liquid water, cloud ice and aerosol for the ensemble of soundings in the simulation. The histograms in blue refer to the whole ensemble; those in red apply after the post-processing filter.
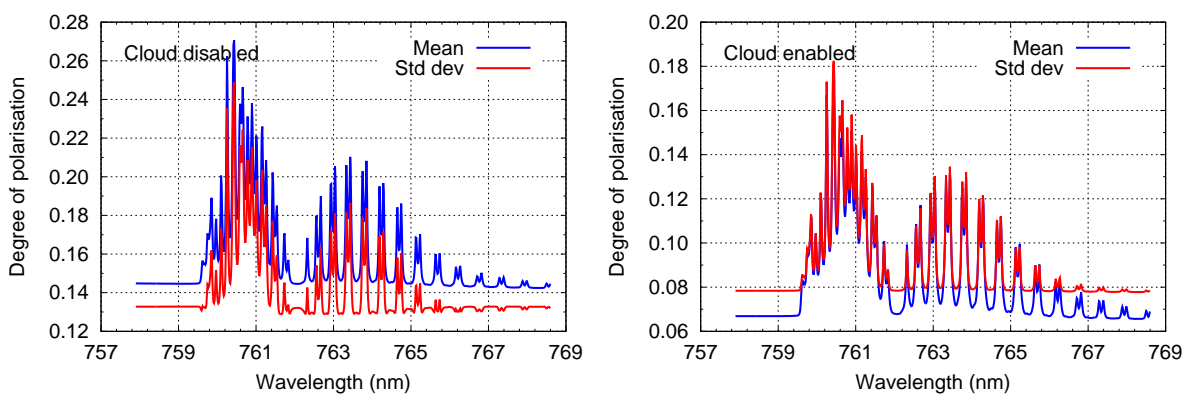

Figure 9. Mean and standard deviation of the degree of polarisation simulated at the top of the atmosphere in the $\mathrm{O}_{2}$ A-band. In the left-hand panel the soundings with cloud were discarded, so the sources of polarisation are the surface and scattering by aerosols and molecules. The right-hand panel applies to soundings with cloud.

\subsection{Experiment 1}

In the first experiment, the measured spectrum was taken to be $I^{\star}$ as defined in Eq. (34). Knowledge of $H$ and $V$ was denied to the retrieval algorithm, so it attempted to match $I^{\star}$ using only the intensity at the top of the atmosphere. Thus, the measured spectrum contains not only the intensity but also the slowly varying wavelength dependence of $(H-V)$, upon which is superimposed the rapid wavelength dependence of $Q_{0}$, while the retrieval algorithm attempts to fit the measured spectrum with the intensity. In a sense this experiment represents the worst case, because it assumes that no pre-flight polarimetric calibration has been performed.

The degree of polarisation, defined by

$\mathcal{P}=\sqrt{Q^{2}+U^{2}+V^{2}} / I$, varies strongly across the absorption spectrum, peaking at the line centres and falling to a backgroud level, determined principally by the surface and Rayleigh scattering, in the continuum between the lines. At wavelengths in the cores of the lines, photons are likely to have been scattered higher in the atmosphere by molecules, clouds and aerosols, which typically have stronger polarisation signatures than the surface. Figure 9 shows the mean and standard deviation of the degree of polarisation in the $\mathrm{O}_{2}$ A-band for the ensemble of soundings in the frames passing through Agra, Wuhan and Alice Springs on the selected days and observation times. In order to illustrate the degree of polarisation likely to be encountered in the almost clear conditions required by the retrieval algorithm, the mean and standard deviation in the left-hand panel of Fig. 9 were computed from the ensemble with cloud disabled. Thus, in this ensemble, polarisation is generated by 

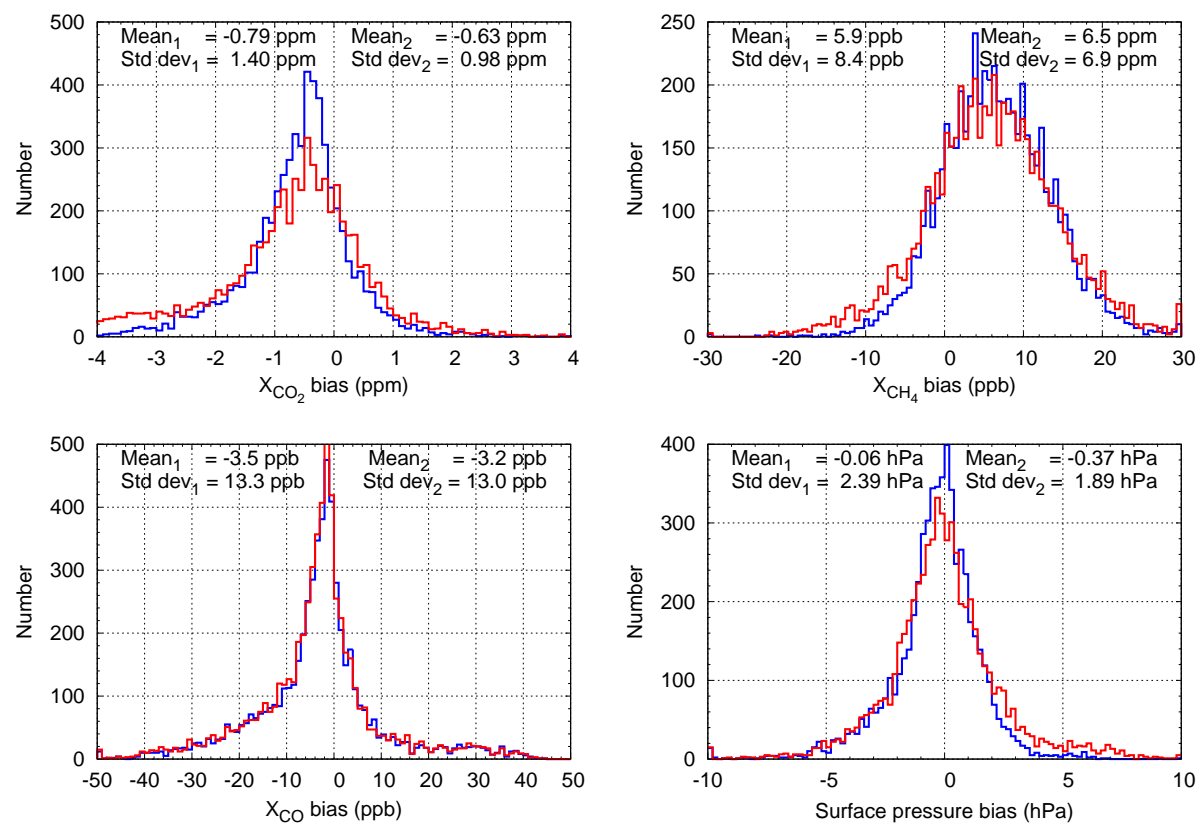

Figure 10. Histograms of the biases in $X_{\mathrm{CO}_{2}}, X_{\mathrm{CH}_{4}}, X_{\mathrm{CO}}$ and surface pressure for the ensemble of soundings in the simulation. The red and blue histograms apply to Experiments 1 and 2, respectively.
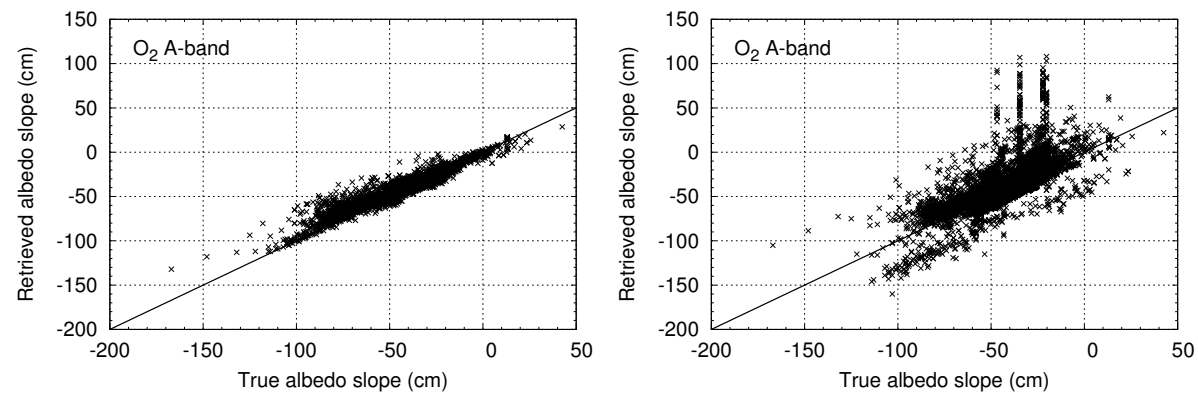

Figure 11. Correlations between true and retrieved albedo slope in the $\mathrm{O}_{2}$ A-band. The slopes (in units $\left(\mathrm{cm}^{-1}\right)^{-1}$ or $\mathrm{cm}$ ) have been multiplied by $10^{6}$. The left-hand panel is for an instrument equipped with ideal polarisation scramblers; the right-hand panel applies to Experiment 2 for geoCARB.

the surface and by scattering from aerosols and molecules, but not from clouds. The right-hand panel applies to the ensemble with cloud enabled.

\subsection{Experiment 2}

In the second experiment, the retrieval algorithm was given access to the instrument Mueller matrix, which for the simplified instrument amounts to knowing the functions $H$ and $V$ derived from the grating efficiencies. Thus, the retrieval algorithm computes $I_{0}+(H-V) Q_{0} / 2$ and uses this to match the measured spectrum. We stress, however, that the retrieval algorithm assumes a non-polarising, Lambertian surface and fixed types of aerosol and cloud whose scattering properties are specified, so its ability to reproduce the measured Stokes vector at the top of the atmosphere is limited.
For reference, the results of this experiment are compared with those from an instrument with an ideal polarisation scrambler, where the measured spectrum is the intensity and the retrieval algorithm attempts to fit the measured spectrum with its internally generated representation of the intensity.

\section{Results}

Histograms of the biases in retrieved $X_{\mathrm{CO}_{2}}, X_{\mathrm{CH}_{4}}, X_{\mathrm{CO}}$ and surface pressure are shown in Fig. 10, while means and standard deviations of the errors are listed in Table 2. For comparison, Table 2 also lists the results obtained for an instrument equipped with ideal polarisation scramblers. The histograms for the case with scramblers are almost indistinguishable from those for Experiment 2, and therefore are not shown. 
The effect of ignoring the polarising properties of the gratings is apparent in the histograms of Fig. 10. The histograms for retrieved $X_{\mathrm{CO}_{2}}$ and surface pressure are broader, with outliers well beyond the targets set for the geoCARB mission. The impact on retrieved $X_{\mathrm{CH}_{4}}$ and $X_{\mathrm{CO}}$ is smaller, for reasons presently unknown, but is still significant. While the differences in the average biases shown in Table 2 appear small, they nevertheless are important, because even small biases on large spatial scales can lead to significant errors in surface fluxes of $\mathrm{CO}_{2}$.

Figure 10 shows that the retrieval algorithm can account for the spectral slope introduced by the gratings, provided that the spectrographs are calibrated before launch. However, there are hidden side effects. For example, the slopes of the surface albedos across the spectral bands of geoCARB, retrieved simultaneously with the gas concentrations, are not as accurate as for the idealised, unpolarised case. This is demonstrated in Fig. 11 for the slope on the $\mathrm{O}_{2}$ A-band albedo. The upper panel shows the correlation between the true and retrieved slopes for the unpolarised case. The correlation is tight, indicating that this parameter is well determined. The lower panel is for Experiment 2 with geoCARB. Although the functions $V(\lambda)$ and $H(\lambda)$ have been supplied to the retrieval algorithm, and although the trace gas concentrations have been retrieved well, there clearly is ambiguity in the slope of the albedo. Because the aim of geoCARB is to retrieve trace gas concentrations, this ambiguity is not a serious concern.

\section{Conclusions}

In this study column-averaged concentrations of $\mathrm{CO}_{2}$ were retrieved from spectra measured at the top of the atmosphere by a geoCARB-like instrument. The ability of the retrieval algorithm to predict the polarisation state is limited because internally it assumes that the surface is non-polarising and Lambertian and that aerosols and clouds are composed from fixed types whose scattering (and polarising) properties are assigned, fixed and usually inconsistent with the real atmosphere. This inability leads to an irreducible minimum error when the algorithm is applied to a realistic ensemble of surfaces and atmospheres.

For an instrument that is sensitive to the degree of polarisation, rather than just to the radiant intensity, the error in retrieved trace gas concentrations is expected to be larger. The reason is that the retrieval algorithm will have difficulty matching the measured spectrum, which is a linear combination of the elements $I, Q, U$ and $V$ of the Stokes vector with coefficients (Stokes coefficients) that are specific to the instrument and the viewing geometry. The Stokes coefficients generally vary slowly with wavelength, though the changes over a band may be large. Thus, the measured spectrum will mix the slow wavelength variation of the Stokes coefficients with the rapid variation inherited from the Stokes compo- nents. Unless the retrieval algorithm can imitate this wavelength dependence, errors in $X_{\mathrm{CO}_{2}}, X_{\mathrm{CH}_{4}}$ and $X_{\mathrm{CO}}$ can be expected.

The experiments in this study show that errors caused by unknown polarisation do arise. However, generally they are small, though they remain significant for $X_{\mathrm{CO}_{2}}$. They are not disastrous because the retrieval algorithm allows the surface albedo to vary linearly with wavelength over each band, and it adjusts the slope during the retrieval. This adjustment of surface albedo with wavelength compensates to a large degree for the wavelength dependence of the Stokes coefficients. Thus, even in the presence of significant polarisation at the entrance aperture, geoCARB should recover reliable estimates for both trace gas concentrations and the bandaveraged surface albedo, but it might assign the slope of the surface albedo incorrectly.

Through radiometric and polarimetric calibration before launch using the procedure defined in this study, errors from polarised surfaces and clouds can be reduced to negligible levels compared with other systematic biases in the retrieval algorithm. If in the future the latter can be reduced, then polarisation biases would need to be re-examined.

Acknowledgements. Polonsky was supported by a contract from Lockheed Martin Advanced Technology Center, Palo Alto, for this work. The retrieval algorithm was adapted by Polonsky from the ACOS algorithm developed for GOSAT by NASA's ACOS team, whose excellent work we gratefully acknowledge. The retrieval algorithm was run on the OCO cluster at Colorado State University, thanks to support from Chris O'Dell. The code that generated geoCARB spectra was written and run in Melbourne on the 16-node cluster at Greenhouse Gas Monitor Australia Pty. Ltd.

Edited by: F. Hase

\section{References}

Deeter, M. N., Emmons, L. K., Francis, G. L., Edwards, D. P., Gille, J. C., Warner, J. X., Khattatov, B., Ziskin, D., Lamarque, J.-F., Ho, S.-P., Yudin, V., Attié, J.-L., Packman, D., Chen, J., Mao, D., and Drummond, J. R.: Operational carbon monoxide retrieval algorithm and selected results for the MOPITT instrument, J. Geophys. Res., 108, 4399, doi:10.1029/2002JD003186, 2003.

Deeter, M. N., Edwards, D. P., and Gille, J. C.: Retrievals of carbon monoxide profiles from MOPITT observations using lognormal a priori statistics, J. Geophys. Res., 112, 11311, doi:10.1029/2006JD007999, 2007a.

Deeter, M. N., Edwards, D. P., Gille, J. C., and Drummond, J. R.: Sensitivity of MOPITT observations to carbon monoxide in the lower troposphere, J. Geophys. Res., 112, 24306 , doi:10.1029/2007JD008929, 2007b.

Heidinger, A. K., O’Dell, C., Bennartz, R., and Greenwald, T.: The successive-order-of-interaction radiative transfer model: Part I: Model development, J. Appl. Meteorol. Clim., 45, 1388-1402, doi:10.1175/JAM2387.1, 2006. 
Kawa, S. R., Erickson, D. J., Pawson, S., and Zhu, Z.: Global $\mathrm{CO}_{2}$ transport simulations using meteorological data from the NASA data assimilation system, J. Geophys. Res., 109, 18312, doi:10.1029/2004JD004554, 2004.

Krol, M., Houweling, S., Bregman, B., van den Broek, M., Segers, A., van Velthoven, P., Peters, W., Dentener, F., and Bergamaschi, P.: The two-way nested global chemistry-transport zoom model TM5: algorithm and applications, Atmos. Chem. Phys., 5, 417432, doi:10.5194/acp-5-417-2005, 2005.

Kumer, J. B., Rairden, R. L., Roche, A. E., Chevallier, F., Rayner, P. J., and Moore, B.: Progress in development of Tropospheric Infrared Mapping Spectrometers (TIMS): geoCARB green house gas (GHG) application, Proc. SPIE, 8867, 88670K, doi:10.1117/12.2022668, 2013.

Mishchenko, M. I., Travis, L. D., and Lacis, A. A.: Scattering, Absorption, and Emission of Light by Small Particles, Cambridge University Press, Cambridge, UK, 2002.

Mobilia, J., Kumer, J. B., Palmer, A., Sawyer, K., Mao, Y., Katz, N., Mix, J., Nast, T., Clark, C. S., Vanbezooijen, R., Magoncelli, A., Baraze, R. A., and Chenette, D. L.: Determination of technical readiness for an atmospheric carbon imaging spectrometer, Proc. SPIE, 8867, 88670L, doi:10.1117/12.2029634, 2013.

Nadal, F. and Breon, F.-M.: Parameterization of surface polarized reflectance derived from POLDER spaceborne measurements, IEEE T. Geosci. Remote, 37, 1709-1718, doi:10.1109/36.763292, 1999.

Nakajima, T. and Tanaka, M.: Algorithms for radiative intensity calculations in moderately thick atmospheres using a truncation approximation, J. Quant. Spectrosc. Ra. 40, 51-69, doi:10.1016/0022-4073(88)90031-3, 1988.

Natraj, V. and Spurr, R. J. D.: A fast linearized pseudo-spherical two orders of scattering model to account for polarization in vertically inhomogeneous scattering-absorbing media, J. Quant. Spectrosc. Ra., 107, 263-293, doi:10.1016/j.jqsrt.2007.02.011, 2007.
O’Brien, D. M., Polonsky, I., O’Dell, C., Kuze, A., Kikuchi, N., Yoshida, Y., and Natraj, V.: Testing the polarization model for TANSO-FTS on GOSAT against clear-sky observations of sunglint over the ocean, IEEE T. Geosci. Remote, 51, 5199-5209, doi:10.1109/TGRS.2012.2232673, 2013.

O’Dell, C., Heidinger, A. K., Greenwald, T., Bauer, P., and Bennartz, R.: The successive-order-of-interaction radiative transfer model: Part II: Model performance and applications, J. Appl. Meteorol. Clim., 45, 1403-1413, doi:10.1175/JAM2409.1, 2006.

O'Dell, C. W.: Acceleration of multiple-scattering, hyperspectral radiative transfer calculations via low-streams interpolation, J. Geophys. Res., 115, D10206, doi:10.1029/2009JD012803, 2010.

Polonsky, I. N., O’Brien, D. M., Kumer, J. B., O’Dell, C. W., and the geoCARB Team: Performance of a geostationary mission, geoCARB, to measure $\mathrm{CO}_{2}, \mathrm{CH}_{4}$ and $\mathrm{CO}$ column-averaged concentrations, Atmos. Meas. Tech., 7, 959-981, doi:10.5194/amt7-959-2014, 2014.

Rayner, P. J., Utembe, S. R., and Crowell, S.: Constraining regional greenhouse gas emissions using geostationary concentration measurements: a theoretical study, Atmos. Meas. Tech., 7, 3285-3293, doi:10.5194/amt-7-3285-2014, 2014.

Sawyer, K., Clark, C., Katz, N., Kumer, J., Nast, T., and Palmer, A.: GeoCARB design maturity and geostationary heritage, Proc. SPIE, 8867, 88670M, doi:10.1117/12.2024457, 2013.

Wiscombe, W. J.: The delta-M method: rapid yet accurate radiative flux calculations for strongly asymmetric phase functions, J. Atmos. Sci., 34, 1408-1422, doi:10.1016/0021-9991(77)90031-6, 1977. 